\title{
Ischemic necrosis of the tongue in surgical patients with septic shock: a case report
}

\author{
Jinbeom Cho, Kiyoung Sung and Dosang Lee
}

\begin{abstract}
Background: As the tongue is a well-vascularized organ, ischemic necrosis of the tongue is a rare disease entity. Critically ill patients with profound shock may experience end-organ hypoperfusion, which might result in tongue necrosis. However, to our best knowledge, there are no reports regarding ischemic necrosis of the tongue in surgical patients with septic shock.

Case presentation: Two patients recently developed ischemic necrosis of the tongue in our surgical intensive care unit. Both patients had undergone emergent surgery for ischemic enteritis and developed postoperative septic shock. The first patient responded to critical treatment with a short period of circulatory shock, and the delivered dose of the vasopressor seemed to be acceptable. In contrast, the second patient developed postoperative refractory shock, and high-dose vasopressor treatment was required to maintain adequate tissue perfusion. Both patients developed ischemic necrosis of the tongue and died shortly after its emergence, despite vigorous resuscitation.

Conclusions: We suggest that ischemic necrosis of the tongue is an under-reported manifestation of any type of circulatory shock, which may have a complex pathogenic mechanism. Clinicians should be aware of the possibility of ischemic necrosis of the tongue in patients with circulatory shock, even if the patient exhibits clinical improvement, as this awareness may facilitate estimation of their prognosis and preparation for clinical deterioration.
\end{abstract}

Keywords: Tongue necrosis, Tongue ischemia, Septic shock, Ischemic enteritis, Case report

\section{Background}

As the tongue is a well-vascularized organ, ischemic necrosis of the tongue is a rare disease entity, which is generally associated with giant cell arteritis (GCA). Critically ill patients with profound shock may experience end-organ hypoperfusion, which might result in tongue necrosis, although this phenomenon has not been widely reported. One group has reported a series of cases with ischemic necrosis of the tongue among patients with cardiogenic shock [1, 2]; however, to our best knowledge, there are no reports of tongue necrosis in surgical patients with septic shock. We suggest that ischemic necrosis of the tongue can develop as a sequela to any type of circulatory shock, although there has been no evidence to support this hypothesis. Two patients recently developed ischemic necrosis of the tongue in our surgical intensive care unit, and we report these

\footnotetext{
* Correspondence: surgeryds@gmail.com

Department of Surgery, Bucheon St. Mary's Hospital, The Catholic University of Korea, College of Medicine, Sosa-dong, Wonmi-gu, Bucheon-siGyunggi-do, (420-717) Korea
}

(c) 2016 The Author(s). Open Access This article is distributed under the terms of the Creative Commons Attribution 4.0 International License (http://creativecommons.org/licenses/by/4.0/), which permits unrestricted use, distribution, and reproduction in any medium, provided you give appropriate credit to the original author(s) and the source, provide a link to the Creative Commons license, and indicate if changes were made. The Creative Commons Public Domain Dedication waiver (http://creativecommons.org/publicdomain/zero/1.0/) applies to the data made available in this article, unless otherwise stated.

\section{Case presentation}

A comparison of the two cases is presented in Table 1.

\section{Patient 1}

An 88-year-old man with hypertension was admitted to the emergency medical center at our hospital based on a complaint of abdominal pain. Abdominal computed tomography (CT) revealed advanced sigmoid colon cancer without any evidence of intestinal perforation or hypoperfusion (Fig. 1). As the patient complained of severe abdominal pain and was hemodynamically unstable, emergent surgery was performed under the suspicion of a surgical emergency. The abdominal cavity was entered through a mid-line incision, and we found ischemic necrosis of the entire colon along with a huge tumor at the sigmoid colon. Therefore, we performed total colectomy with ileorectal anastomosis. There was no evidence of occlusion in the major mesenteric vessels, including the 
Table 1 Comparison between the two cases

\begin{tabular}{|c|c|c|}
\hline & Patient 1 & Patient 2 \\
\hline Age (years) & 88 & 80 \\
\hline Gender & Male & Female \\
\hline Clinical presentation & Septic shock & Septic shock \\
\hline Intraoperative diagnosis & Ischemic necrosis of the colon & Ischemic necrosis of the ileum \\
\hline Operation & Total colectomy & Segmental resection of the ileum \\
\hline Possible cause of mesenteric ischemia & unknown & femoral herniation of ileum \\
\hline Length of vasopressor treatment ${ }^{a}$ (days) & 3 & until death \\
\hline Length of mechanical ventilation ${ }^{a}$ (days) & 5 & until death \\
\hline Length of CRRT ${ }^{\mathrm{a}}$ (days) & until death & until death \\
\hline Maximum dose of norepinephrine ( $\mu \mathrm{g} / \mathrm{kg} / \mathrm{min})$ & 0.5 & 2 \\
\hline Maximum dose of epinephrine $(\mu \mathrm{g} / \mathrm{kg} / \mathrm{min})$ & 0.05 & 0.5 \\
\hline \multicolumn{3}{|l|}{ Coagulation profile ${ }^{b}$} \\
\hline Antithrombin III (\%) & 28 & 13 \\
\hline Fibrinogen (mg/ml) & 201 & 97 \\
\hline $\mathrm{FDP}(\mu \mathrm{g} / \mathrm{ml})$ & 8.6 & 18.7 \\
\hline SOFA score at ICU admission ${ }^{b}$ & 13 & 16 \\
\hline Respiratory $\left(\mathrm{PaO}_{2} / \mathrm{FiO}_{2}\right)$ & 200 & 150 \\
\hline Coagulation (Platelet, $\times 10^{3} / \mu \mathrm{L}$ ) & 80 & 40 \\
\hline Liver (Total bilirubin, mg/dL) & 1.6 & 3.4 \\
\hline Dose of norepinephrine ( $\mu \mathrm{g} / \mathrm{kg} / \mathrm{min})$ & 0.5 & 1 \\
\hline Glasgow Coma Scale score & 13 & 13 \\
\hline Renal system (Creatinine, mg/dL) & 2.7 & 3.6 \\
\hline Blood culture test ${ }^{\mathrm{b}}$ & Candida species & none \\
\hline Time to enteral feeding & 5th POD & none \\
\hline Time to necrosis of the tongue & 7th POD & 8th POD \\
\hline Pressure on the tongue from endotracheal tube & none & suspicious \\
\hline Concomitant signs of other organ hypoperfusion & none & Lower limb \\
\hline Outcome & Died on the 12 nd POD & Died on the 10th POD \\
\hline
\end{tabular}

CRRT continuous renal replacement therapy, FDP fibrin-degradation product, SOFA Sepsis-Related Organ Failure Assessment Score, ICU intensive care unit, POD post-operative day

includes both the day of operation and the day of complete cessation

${ }^{b}$ checked immediately after the operation

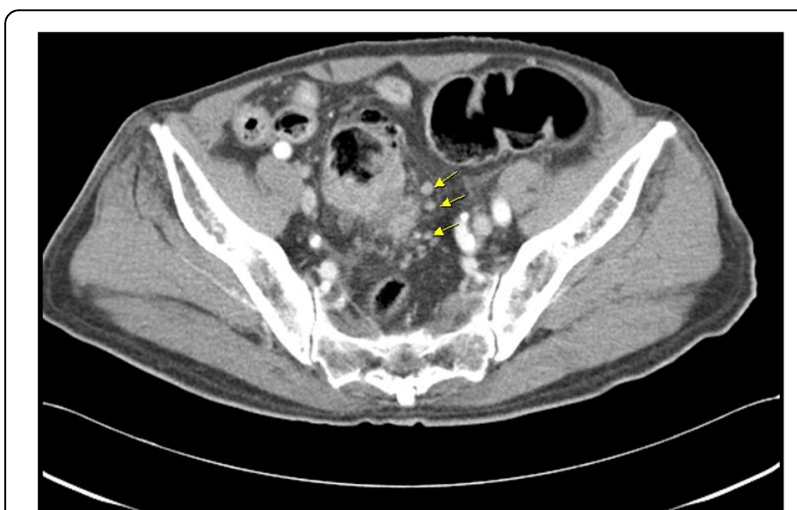

Fig. 1 Computed tomography of abdomen shows huge sigmoid colon cancer. Arrows indicate enlarged peri colic lymph node celiac trunk, superior mesenteric artery, and inferior mesenteric artery. After the surgery, we administered critical care for shock reversal and maintenance of adequate organ perfusion. The coagulation profile of Patient 1 suggested disseminated intravascular coagulation (DIC) (Table 1). On the second postoperative day (POD), we ceased the vasopressor and inotropic treatment, and observed that adequate blood pressure was maintained. We weaned the patient off the mechanical ventilation and performed extubation on the fourth POD, and enteral feeding was started via a nasogastric tube on the fifth POD. The patient responded well to the treatment, and we proceeded to the de-escalation phase (with negative fluid balance) of the intensive treatment. However, we noted a darkish coloring of the 
patient's tongue on the seventh POD (Fig. 2), and the laboratory findings began to deteriorate (newly noted band neutrophils, decreasing platelet counts, and increasing lactate levels). Because the endotracheal tube had been removed on the fourth POD, it was unlikely that the discoloration was due to the pressure from the endotracheal tube on the tongue. Furthermore, there was no evidence of hypoperfusion in other organs, such as in the limbs or intestines. We performed bed-side duplex ultrasonography and did not detect any occlusive lesions in the external carotid or facial arteries. However, both lingual arteries arising from the external carotid arteries were not visible during the duplex examination. Additionally, bed-side laryngoscopy revealed no mucosal injury in the oral cavity or on the tongue. A meticulous oral hygiene regimen was started, and we administered empirical antiplatelet therapy. However, Patient 1 became hemodynamically unstable and developed multi organ failure on the eighth POD, and subsequently died on the twelfth POD, despite vigorous resuscitation.

\section{Patient 2}

An 80-year-old woman underwent herniorraphy for an incarcerated left femoral hernia and there was no evidence of intestinal perforation or hypoperfusion. On the next day, she complained of severe abdominal pain and exhibited profound shock. Abdominal CT scan revealed intraperitoneal free air and decreased perfusion of the small intestine (Fig. 3). Emergent surgery was performed and the findings revealed perforation of the ileum and ischemic necrosis of the small intestine. There was no evidence of major mesenteric vessel occlusion and we resected approximately $20 \mathrm{~cm}$ of the necrotic small intestine. After the surgery, the patient was admitted to the surgical intensive care unit for postoperative care, with a clinical presentation of septic shock, severe

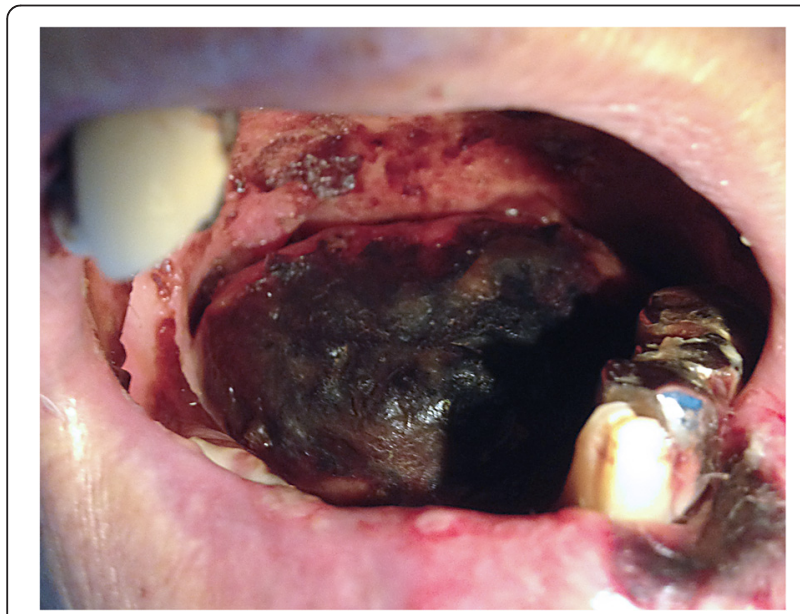

Fig. 2 Bilateral gangrene of the oral tongue

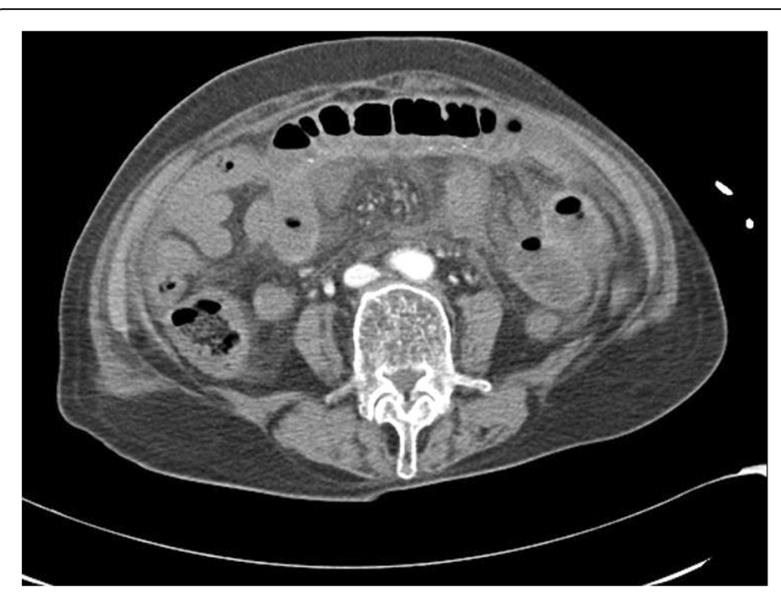

Fig. 3 Computed tomography of abdomen shows intraperitoneal free air and decreased perfusion of the small intestine

hypotension, respiratory failure, and anuria. Therefore, we started high-dose vasopressor and inotropic treatment, broad-spectrum antibiotics, fluid resuscitation, mechanical ventilation, and continuous renal replacement therapy to maintain adequate blood pressure and organ perfusion. As shown in Table 1, the coagulation profile of Patient 2 also suggested DIC. We could not taper the intensive treatment because the patient exhibited persistent shock. The highdose vasopressor treatment (epinephrine at $0.5 \mu \mathrm{g} / \mathrm{kg} / \mathrm{min}$, norepinephrine at $2 \mu \mathrm{g} / \mathrm{kg} / \mathrm{min}$, and no vasopressin) was maintained for 5 days, and tapering was started on the sixth POD. We observed a necrotic change at the tongue on the eighth POD (Fig. 4), as well as critical lower limb ischemia and a worsening in the patient's general condition. Thus, we performed bed-side laryngoscopy and duplex ultrasonography; however, these examinations did not reveal any diagnostic clues. Unfortunately, we were unable to perform more invasive evaluations (e.g., angiography or biopsy), due to the patient's poor general condition. We considered tracheostomy, although the patient and her family refused the procedure, and she

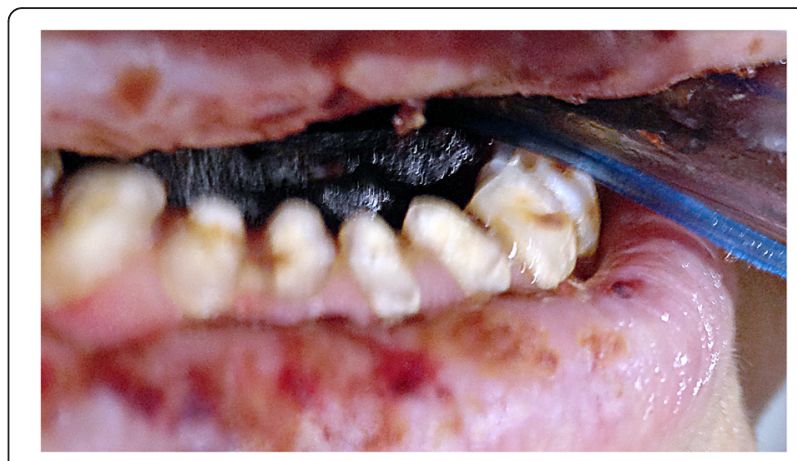

Fig. 4 Bilateral gangrene of the oral tongue. The endotracheal tube was placed 
subsequently died two days later from multi-organ failure. Enteral feeding could not be delivered and mechanical ventilation was performed until the end of the treatment.

\section{Discussion}

Ischemic necrosis of the tongue has occasionally been reported, and is mainly associated with GCA [2]. GCA, or temporal arteritis, is a systemic granulomatous arteritis that involves medium and large arteries, especially branches from the aortic arch, and is mainly found in older women [3]. The cause of this disease is unknown, although it may be an immune-mediated condition [4]. The clinical manifestations of GCA include headache, ocular symptoms, masseteric pain, and tongue pain [3], and systemic corticosteroid therapy is known to be effective for ameliorating the symptoms of GCA. Lingual arteritis can develop in 25 of the patients with GCA, and $15 \%$ of these patients experience lingual artery infarction [4]. In cases of GCA, tongue necrosis may develop secondary to the lingual arteritis, and is typically unilateral, with the exception of one reported case of bilateral tongue necrosis $[1,5]$. The diagnosis of GCA usually depends on a clinical suspicion, and arterial biopsy can be performed for unclear cases. The laboratory findings in cases of GCA may include an elevated erythrocyte sedimentation rate, elevated plasma fibrinogen levels, and elevated $\alpha-2$ globulin levels [4]. Other known causes of tongue necrosis include Kawasaki disease, Wegener's granulomatosis, DIC, and essential thrombocytosis, although these cases are rarely reported [1].

Tongue necrosis was first reported as a sequela of shock in 2007 [2]. The same group also reported a case series of tongue necrosis that was associated with cardiogenic shock in 2010 [1], and they suggested that impaired perfusion of the tongue in cases of severe shock appeared to be the most reasonable explanation. However, relative blood flow to the heart increases during circulatory shock, and brain perfusion is maintained. Furthermore, the tongue is well supplied by the bilateral lingual arteries and branches of the facial and pharyngeal arteries. Therefore, it is difficult to explain how decreased blood flow to the tongue could occur without concomitant hypoperfusion of the gut, skin, and musculoskeletal system. However, Roman et al. have presented a hypothesis that could explain the hypoperfusion of the tongue during severe shock: blood flow to the internal carotid artery might be protected at the expense of the external carotid system during periods of decreased circulatory volume, and this would reduce the supply of blood to the tongue, which is a potentially susceptible muscular end organ [1].

A search of the literature reveals several other potential causes of tongue necrosis, such as the prolonged use of terlipressin (an anti-diuretic hormone analogue) [6] and DIC [7]. However, it remains unclear what severity of DIC, or what dose of vasopressor treatment, can cause tongue necrosis. As shown in Table 1, the two patients in this report experienced different clinical courses. Patient 1 responded to the critical care with a short period of circulatory shock, and the delivered dose of the vasopressor seemed to be adequate. In contrast, Patient 2 experienced postoperative refractory shock, which necessitated high-dose vasopressor treatment to maintain adequate tissue perfusion. Moreover, Patient 1 did not exhibit any concomitant signs of hypoperfusion to other organs. Therefore, we cautiously suggest that there might be no specific vasopressor dose or severity of shock that must be exceeded to cause ischemic necrosis of the tongue. If the hypothesis that vasopressor or circulatory shock might cause tongue necrosis is correct, other contributing factors are likely needed to induce ischemic necrosis of the tongue during shock. Our cases, and the other reported cases [1], exhibited bilateral tongue necrosis with rapid progression, and the necrosis progressed from the distal section to the proximal section of the tongue, which was less consistent with local pressure from an endotracheal tube and more consistent with a systemic process. This feature was also different from the unilateral necrosis that is observed in cases of GCA, which is a slowly progressing immune disease. Thus, it is possible that no single factor can cause bilateral lingual artery infarction in patients with circulatory shock, and the tongue necrosis may be promoted by complex interactions between the decreased circulatory volume, DIC, vasoconstrictor use, and pressure on the tongue from the endotracheal tube. Therefore, we suggest that tongue necrosis might be induced by any severity of shock, and at any time during the shock treatment, if the interaction(s) between these contributing factors is triggered. Moreover, ischemic necrosis of the tongue might predict a poor prognosis, despite apparent improvements in the other manifestations of shock.

We noticed that our patients developed septic shock secondary to gangrenous necrosis of the intestine. According to the intraoperative findings, Patient 1 seemed to have developed nonocclusive mesenteric ischemia, because we observed no occlusion of the major mesenteric vessels or other causes of ischemic enteritis. This disease entity is considered the end result of the physiological response to a decreased intravascular volume, and the splanchnic vasoconstriction can exhibit a profound and early onset, even before systemic hemodynamic instability arises [8, 9]. Therefore, undetected circulatory compromise in Patient 1 might have proceeded before the gangrenous necrosis of the intestine and septic shock eventually occurred. We hypothesize that the lingual artery infarction might have already developed and that critical end-organ damage at the tongue had progressed, similar to the pathogenesis of the nonocclusive 
mesenteric ischemia, despite the manifestations of septic shock improving after the operation. However, this hypothesis cannot be applied to Patient 2, because her intestinal necrosis seemed to be caused by mechanical herniation of the intestine.

\section{Conclusion}

In conclusion, we suggest that ischemic necrosis of the tongue is an under-reported manifestation of any type of circulatory shock. The condition may have a complex pathological mechanism, which might consist of interactions between several contributing factors, such as a decreased intravascular volume, DIC, vasopressor treatment, and mechanical pressure from the endotracheal tube. Furthermore, it is possible that no single factor can cause tongue necrosis. Therefore, clinicians should be aware of the possibility of ischemic necrosis of the tongue in patients with circulatory shock, even if they exhibit clinical improvement, as this awareness may facilitate estimation of their prognosis and preparation for clinical deterioration.

\section{Abbreviations}

$C T$, computed tomography; DIC, disseminated intravascular coagulation; GCA, giant cell arteritis; POD, postoperative day

\section{Acknowledgements}

The authors thank to our surgical residents and surgical critical nursing team for their sincere assistance in surgery and post-operative management.

\section{Funding}

None.

\section{Authors' contributions}

$J C$ and DL participated in surgical treatment and prepared manuscript and performed the literature search; KS participated in manuscript revision and final review. All authors read and approved the final manuscript.

\section{Competing interests}

The authors declare that they have no competing interests.

\section{Consent for publication}

Written informed consents were obtained from the patients' family for publication of these two Case Reports and any accompanying images.

\section{Ethics approval and consent to participate}

Not applicable.

Received: 16 February 2016 Accepted: 13 July 2016

Published online: 19 July 2016

\section{References}

1. Roman BR, Immerman SB, Morris LG. Ischemic necrosis of the tongue in patients with cardiogenic shock. Laryngoscope. 2010;120:1345-9.

2. Morris LG, Komisar A, Liberatore LA. Total gangrene of the oral tongue following intra-aortic balloon pump for cardiogenic shock. Otolaryngol Head Neck Surg. 2007;137:358-9.

3. Patterson A, Scully C, Barnard N, Griffiths MJ, Eveson JW, Novelli M. Necrosis of the tongue in a patient with intestinal infarction. Oral Surg Oral Med Oral Pathol. 1992;74:582-6.

4. Llorente Pendás S, De Vicente Rodríguez JC, González García M, Junquera Gutierrez LM, López Arranz JS. Tongue necrosis as a complication of temporal arteritis. Oral Surg Oral Med Oral Pathol. 1994;78:448-51.

5. Missen GAK. Gangrene of the tongue. Br Med J. 1961;1:1393-4.
6. Mégarbané $H$, Barete $S$, Khosrotehrani $K$, Izzedine $H$, Moguelet $P$, Chosidow $\mathrm{O}$, Frances $\mathrm{C}$, Aractingi $\mathrm{S}$. Two observations raising questions about risk factors of cutaneous necrosis induced by terlipressin (Glypressin). Dermatology. 2009;218:334-7.

7. Kamatani T, Yamashita K, Okabayashi T, Maeda H, Toi M, Yamamoto T. Bilateral ischemic necrosis of the tongue due to disseminated intravascular coagulation. Int J Oral Maxillofac Surg. 2008;37:777-9.

8. Toung T, Reilly PM, Fuh KC, Ferris R, Bulkley GB. Mesenteric vasoconstriction in response to hemorrhagic shock. Shock. 2000;13:267-73.

9. Hamilton-Davies C, Mythen MG, Salmon LB, Jacobson D, Shukla A, Webb AR. Comparison of commonly used clinical indicators of hypovolaemia with gastrointestinal tonometer. Intensive Care Med. 1997;23:276-81.
Submit your next manuscript to BioMed Central and we will help you at every step:

- We accept pre-submission inquiries

- Our selector tool helps you to find the most relevant journal

- We provide round the clock customer support

- Convenient online submission

- Thorough peer review

- Inclusion in PubMed and all major indexing services

- Maximum visibility for your research

Submit your manuscript at www.biomedcentral.com/submit 